\title{
Periodontitis and rheumatoid arthritis: A neglected association in developing countries
}

\author{
Nasibeh Alebooyeh ${ }^{1}$, Jamileh Moghimi ${ }^{* 2}$, Amir Hasani ${ }^{3}$, Raheb Ghorbani ${ }^{4}$, Golmehr Taheri $^{5}$ \\ ${ }^{1}$ Faculty of Dentistry, Semnan University of Medical Sciences, Semnan, Iran. ${ }^{2}$ Social Determinants of Health Re- \\ search Center, Semnan University of Medical Sciences, Semnan, Iran. ${ }^{3}$ Department of Internal Medicine, Semnan \\ University of Medical Sciences, Semnan, Iran. ${ }^{4}$ Social Determinants of Health Research Center, Semnan University of \\ Medical Sciences, Semnan, Iran. ${ }^{5}$ Department of Internal Medicine, Semnan University of Medical Sciences, Semnan, \\ Iran
}

Most patients with rheumatoid arthritis (RA), one of the most common inflammatory diseases, also suffer from periodontitis. This study investigated the effects of periodontal therapy on disease activity in RA patients.

In this before-and-after clinical trial study, 30 patients with simultaneous RA and periodontitis were studied from March 2014 to February 2016. The Disease Activity Score with 28-joint Counts (DAS28) questionnaire was completed for all participants. Patients with confirmed periodontitis received non-surgical scaling, root planning, systemic antibiotics, and chlorhexidine. At days 45 and 90 after treatment, the DAS28, erythrocyte sedimentation rate (ESR), and C-reactive protein (CRP) were measured again. The results of the evaluation of 30 patients with a mean age of $50.2 \pm 10.62$ years ( $80 \%$ female) showed that the difference in the level of disease activity between baseline versus $45(5.18 \pm 0.092$ vs. $4.02 \pm 1.22, P$ value $<0.001)$ and 90 days later $(5.18 \pm 0.092$ vs. $3.84 \pm 0.92, P$ value $<0.001)$, was statistically significant. Serum CRP levels $(\mathrm{mg} / \mathrm{dl})$ were lower at 90 days after treatment $(5.17 \pm 2.96)$ than at 40 days post-treatment (5.30 \pm 3.59$)$. The ESR values (mm/hour) at $45(20.57 \pm 11.01)$ and $90(18.23 \pm 8.99)$ days after periodontal therapy were lower in comparison with the baseline $(21.67 \pm 11.65)$, but there were no significant differences between the different time points $(P$ value $>0.05)$.

Treating periodontitis resulted in reduced RA disease activity. Hence, routine dental examinations for faster diagnosis and treatment of periodontitis in order to relieve RA symptoms as well as further studies including a control group are recommended.

Keywords: Rheumatoid arthritis, periodontitis, periodontal treatment

\section{Introduction}

Rheumatoid arthritis (RA) is a chronic inflammatory systemic disease with unknown etiology and characterized by the involvement of multiple peripheral joints (polyarthritis) $[1,2]$. About $0.5 \%$ to $1 \%$ of the adult populations of the world are suffering from RA; the incidence rate is 2 to 3 times higher in women than in men [1, 3-7].

In spite of its uncertain etiology, genetic and environmental factors such as age, sex, smoking, genetic predisposition, hormonal status, nutrition, socio-economic status, and racial factors have been considered effective in causing or worsening RA [8,9]. One of these factors is Porphyromonas gingivalis, which contains the enzyme peptidyl-arginine deiminase. This enzyme allows bacteria to produce citrullinated peptides [10]. The presence of the peptides causes intolerance to endogenous citrullinated antigens, thereby resulting in the production of anti-citrullinated peptide/protein antibodies (ACPA) that take part in the formation of RA.P. gingivalis and also activate other proteins that are involved in this process $[11,12]$. The bacterium, with its role in RA, is the leading cause of periodontal diseases [12-15].

Periodontal disease usually begins with gingivitis followed by loss of supporting connective tissue such as the alveolar bone, and thus, the separation of the periodontal ligament around the tooth from the cementum [13-15]. Clinical manifestations include increased probing depth, bleeding on probing, increased tooth mobility, and ultimately tooth loss [16].

RA and periodontitis show common pathological fea-

Personal non-commercial use only.Rheumatology Research Journal. Copyright (C) 2019. All rights reserved

*Corresponding Author: Jamileh Moghimi, Social Determinants of Health Research Center, Semnan University of Medical Sciences, Semnan, Iran. Email: moghimi_jam@yahoo.com

Received: 25 April 2018 ; Accepted: 15 October 2018 
tures: 1) both are chronic and inflammatory diseases influenced by genetic and environmental factors as well as the immune imbalance of regulatory factors, and 2) both lead to the destruction of connective and hard tissue [17, 18]. Dental plaque, tooth loss, increased periodontal pocket depth, and loss of tooth attachment are common in RA patients [18].

Due to the limited number of studies on the influence of periodontitis therapy on RA and the lack of a comprehensive study in Iran, the present study was carried out to evaluate the impact of nonsurgical periodontitis treatment on disease activity in rheumatoid arthritis patients.

\section{Materials and Methods}

\section{Patients}

In this before-and-after clinical trial study, 604 established RA patients matching the inclusion criteria were consecutively recruited between March 2014 and February 2016 at the outpatient clinic of rheumatology at the University of Medical Sciences, Semnan, and selected using the census method. The inclusion criteria included age $\geq 18$ years, RA duration $\geq$ one year, RA disease activity number (DAS-28) between 3.2 and 5.1, no change in type or dose medication in three months prior to the first examination (baseline), having more than six natural teeth, and literacy. None of the patients were on biological therapies.

Individuals at risk of infection; inflammatory diseases; severe hepatic or renal impairment; uncontrolled diabetes; frequent use of antibiotics or anti-thrombotic; sensitivity to chlorhexidine digluconate; amoxicillin and metronidazole contraindications; or pregnancy, lactation, or planned pregnancy within the 90-day period of this study were excluded from the research.

\section{Ethics approval}

This study was conducted with the approval of the Medical Ethical Committee of the Semnan University of Medical Sciences, Iranian Registry of Clinical Trials (IRCT2015122512823N3), and all participants provided written informed consent prior to study enrollment according to the Declaration of Helsinki (General Assembly, October 2008).

\section{Clinical examination of rheumatoid arthritis}

After obtaining ethical approval and patients' satisfaction, the Disease Activity Score with 28-joint Counts (DAS28) and demographic questionnaires were completed for all subjects. The DAS2 8 is a questionnaire designed to evaluate the activity of RA using the number of joints with pain or swelling, erythrocyte sedimentation rate (ESR), and general health of the patient using the visual analogue scale (VAS) $[19,20]$. DAS28 is continuous and has a Gaussian distribution with a theoretical range from 0 to 10 . DAS28 values $\leq 2.5$ are regarded as "remission",
2.6-3.1 are regarded as "low disease activity," 3.2-5.0 are regarded as "moderate disease activity," and values $\geq 5.1$ are regarded as"high disease activity" [21]. The selected patients were in the "moderate disease activity" category.

\section{Clinical examination of periodontitis}

Patients were examined by a registered dentist using the Dutch Periodontal Screening Index (DPSI) and 6 sites per tooth (vestibule, distobuccal, mesio-buccal, lingual, disto-lingual, and mesio-lingual) were recorded. The DPSI is a validated index based on bleeding upon probing, pocket probing depth, and clinical attachment loss; patients were categorized as having no periodontitis, moderate periodontitis, or severe periodontitis. Patients with moderate periodontitis were selected for this study.

\section{Interventions and follow ups}

From 85 patients with RA, 30 of them had both RA and periodontitis (Figure 1). Patients were examined by a registered dentist. Upon confirmation of periodontitis, they underwent treatments including non-surgical scaling, root planing, systemic antibiotics (10-day period, capsules of amoxicillin and metronidazole, 3 per day), and chlorhexidine. Root planing was performed under local anesthesia with Gracey curettes (Hu-Friedy, Chicago, IL). Oral health education was also given to the patients. On days 45 and 90 , the patients were again examined by a rheumatologist, and the DAS28 questionnaires were completed. The ESR and CRP at each visit were evaluated in the reference laboratories at Semnan University of Medical Sciences.

\section{Statistical analysis}

Data was analyzed with the SPSS18 software. One-way ANOVA repeated measure (to adjust for multiple comparisons, the Bonferroni test was used), paired $t$, or Wilcoxon tests were used to compare the differences between baseline and days 45 and 90 after periodontal therapy within the same group. A value of $P$ value $<0.05$ was considered statistically significant. 


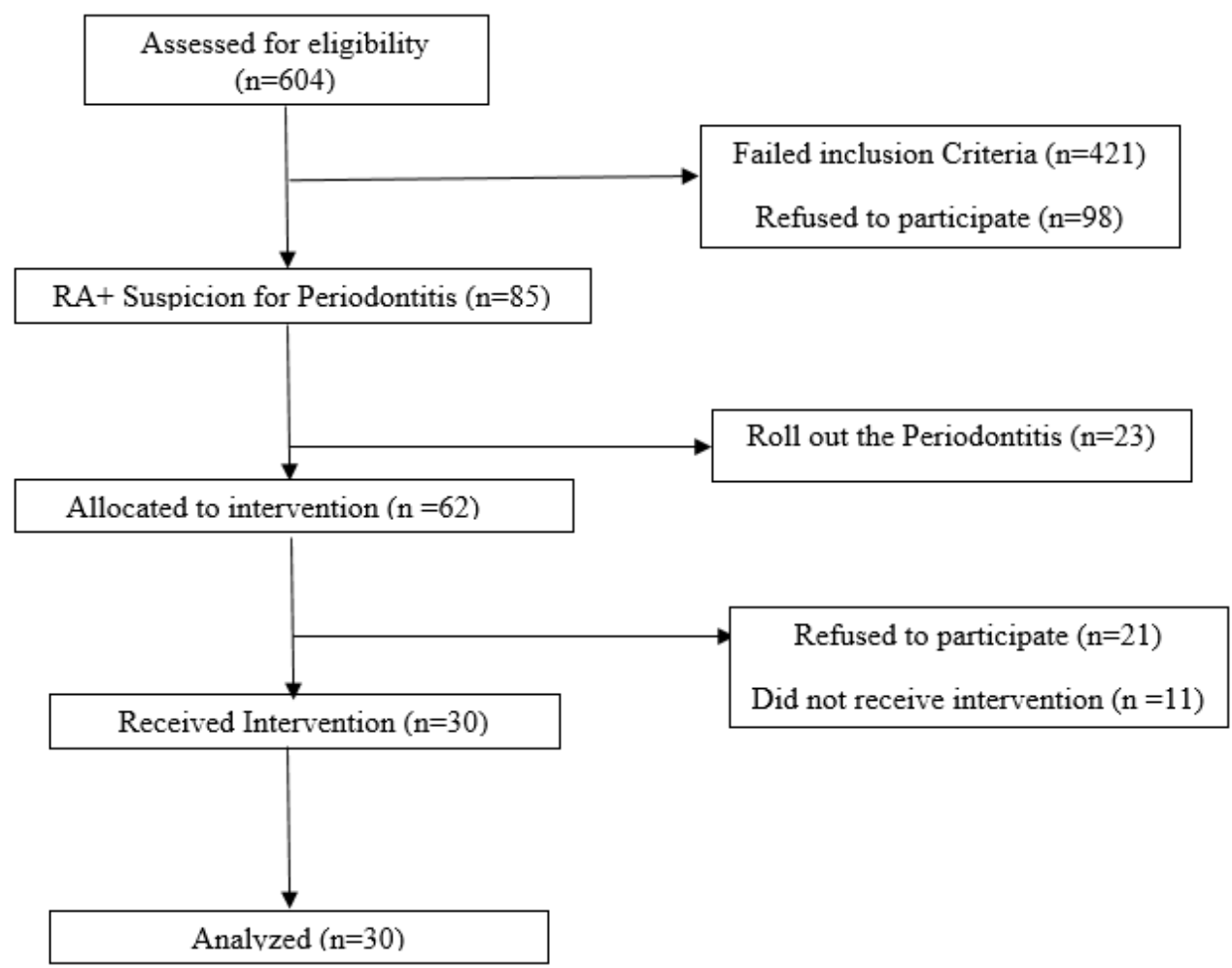

Figure 1. Flow diagram

\section{Results}

In this study, 30 patients had both RA and periodontitis. A total of 30 patients with a mean age of $50.20 \pm 10.62$ years, weight of $70.50 \pm 11.42 \mathrm{~kg}$, and body mass index (BMI) of $31.4 \pm 15.27 \mathrm{~kg} / \mathrm{m} 2$ were enrolled into this study. Table 1 shows other demographic information. The distribution of RA activity levels according to the DAS28 values at different time points, following nonsurgical periodontal therapy, is shown in Table 2. The RA activity level and laboratory markers related to RA status (ESR and CRP) at different time points, following nonsurgical periodontal therapy, are shown in Tables 3 and 4. According to these tables, the difference between the baseline value and day 45 in CRP $(4.77 \pm 3.71$ vs. $5.30 \pm 3.59, P$ value $=0.497)$ and ESR $(21.67 \pm 11.65$ vs. $20.57 \pm 11.01, P$ value $=0.683)$ was not statistically significant.

Table 1. Demographic variables in the study group $(n=30)$

\begin{tabular}{lccc}
\hline & Charateristics & n & \% \\
\hline \multirow{3}{*}{ Age } & early adulthood (20-39) & 4 & 13.3 \\
& middle adulthood (40-59) & 20 & 66.7 \\
Gender & old age $(\geq 60)$ & 6 & 20.0 \\
& female & 24 & 80.0 \\
BMI & male & 6 & 20.0 \\
& underweight $(<18.5)$ & - & - \\
& normal weight (18.5-24.9) & 10 & 33.3 \\
Marriage & overweight $(25-29.9)$ & 13 & 43.4 \\
& obesity $(\geq 30)$ & 7 & 23.3 \\
& married & 29 & 96.7 \\
\hline
\end{tabular}




\begin{tabular}{lccc}
\hline \multicolumn{2}{c}{ Charateristics } & n & \% \\
\hline \multirow{4}{*}{ Job } & Self-employed & 1 & 3.3 \\
& homemaker & 19 & 63.4 \\
& employee & 5 & 16.7 \\
& worker & 1 & 3.3 \\
& other & 4 & 13.3 \\
& $<12$ & 2 & 6.7 \\
Duration of RA & $12-24$ & 8 & 26.7 \\
(month) & $25-60$ & 5 & 16.6 \\
& $61-120$ & 7 & 23.3 \\
& $\geq 121$ & 8 & 26.7 \\
\hline
\end{tabular}

Table 2. Distribution [N (percent)] of the patients with different disease activity of RA at baseline, at 45 and 90 days following nonsurgical periodontal therapy

\begin{tabular}{lccc}
\hline \multicolumn{1}{c}{ Disease activity level (DAS28 Score) } & Baseline $(\mathbf{n = 3 0 )}$ & $\mathbf{4 5}$ days $(\mathbf{n}=\mathbf{3 0})$ & $\mathbf{9 0}$ days $(\mathbf{n}=\mathbf{3 0})$ \\
\hline Remission $(\leq 2.5)$ & - & $3(10.0 \%)$ & $1(3.3 \%)$ \\
Low activity $(2.6-3.1)$ & - & $3(10.0 \%)$ & $8(26.7 \%)$ \\
Moderate activity $(3.2-5.0)$ & $11(36.7 \%)$ & $14(46.7 \%)$ & $12(40.0 \%)$ \\
$(1.5 \leq)$ High activity & $19(63.3 \%)$ & $10(33.3 \%)$ & $9(30.0 \%)$ \\
\hline
\end{tabular}

Table 3. Mean \pm Standard deviation of parameters at baseline, 45 and 90 days following non-surgical periodontal therapy

\begin{tabular}{lccc}
\hline \multicolumn{1}{c}{ Parameters } & Baseline $(\mathbf{n}=\mathbf{3 0})$ & $\mathbf{4 5}$ days $(\mathbf{n}=\mathbf{3 0})$ & 90 days $(\mathbf{n}=\mathbf{3 0})$ \\
\hline CRP (mg/dl) & $4.77 \pm 3.71$ & $5.30 \pm 3.59$ & $5.17 \pm 2.96$ \\
ESR (mm/hour) & $21.67 \pm 11.65$ & $20.57 \pm 11.01$ & $18.23 \pm 8.99$ \\
$\begin{array}{l}\text { Disease activity level } \\
\text { (DAS28) }\end{array}$ & $5.18 \pm 0.92$ & $4.02 \pm 1.22$ & $3.84 \pm 0.92$ \\
\hline
\end{tabular}

The difference between the baseline value and day 90 in $\mathrm{CR} P(4.77 \pm 3.71$ vs. $5.17 \pm 2.96, P$ value $=0.589)$ and $\mathrm{ESR}$ $(21.67 \pm 11.65$ vs. $18.23 \pm 8.99$, and $P$ value $=0.116)$ was not statistically significant. Also, the difference between days 45 and 90 in $\operatorname{CRP}(5.30 \pm 3.59$ vs. $5.17 \pm 2.96, P$ value $=0.753)$, ESR $(20.57 \pm 11.01$ vs. $18.23 \pm 8.99, P$ val$\mathrm{ue}=0.164)$ and the level of disease activity $(4.02 \pm 1.22 \mathrm{vs}$. $3.84 \pm 0.92, P$ value $=0.190)$ was not statistically significant. The ESR values at 45 and 90 days after periodontal therapy were lower in comparison with the baseline, but there were no significant differences between the different time points $(P$ value $>0.05)$. The serum CR $P$ levels were lower at 90 days after non-surgical periodontal therapy than at 40 days, but there were no significant differences between the different time points ( $P$ value $>0.05)$.

One-way ANOVA repeated measure showed that mean disease activity levels (DAS28) among baseline, day 45, and day 90 were significant $\left(P\right.$ value $<0.001, \mathrm{R}^{2}=0.55$, observed power $=1.00$ ).

The differences between the disease activity at baseline versus day $45(5.18 \pm 0.092$ vs. $4.02 \pm 1.22, P$ value $<0.001)$ and at baseline versus day 90 ( $5.18 \pm 0.92$ vs. $3.85 \pm 0.92$, $P$ value $<0.001)$ were statistically significant, but the difference between day 45 and day 90 was not significant $(P$ value $=0.570)($ Tables 3 and 4$)$. In more accurate means, disease activity levels (DAS28) were significantly lower at 90 and 45 days after non-surgical periodontal therapy compared with baseline $(P$ value $<0.05)$. In comparison, the difference in disease activity between baseline and day 45 ( $P$ value $<0.001)$, baseline and day $90(P$ value $<0.001)$ was found to be statistically significant using the Wilcoxon test. 
Table 4. Paired t-test analyses for parameters at baseline, 45 and 90 days following non-surgical periodontal therapy

\begin{tabular}{|c|c|c|c|c|}
\hline \multirow{2}{*}{ Parameters } & \multirow{2}{*}{ Time } & \multicolumn{2}{|c|}{$\begin{array}{l}\text { 95\% Confidence Interval of } \\
\text { the Difference }\end{array}$} & \multirow{2}{*}{ *p-value } \\
\hline & & Lower & Upper & \\
\hline \multirow{3}{*}{ CRP (mg/dl) } & Baseline $* 45$ days & -2.120 & 1.053 & 0.497 \\
\hline & Baseline * 90 days & -1.896 & 1.096 & 0.589 \\
\hline & days $* 90$ days 45 & -0.726 & 0.992 & 0.753 \\
\hline \multirow{3}{*}{ ESR (mm/hour) } & Baseline $* 45$ days & -4.350 & 6.550 & 0.683 \\
\hline & Baseline $* 90$ days & -0.907 & 7.773 & 0.116 \\
\hline & days $* 90$ days 45 & -0.007 & 5.674 & 0.164 \\
\hline \multirow{3}{*}{ DAS28 (score) } & Baseline $* 45$ days & 0.770 & 1.539 & $0.001>$ \\
\hline & Baseline $* 90$ days & 0.946 & 1.716 & $0.001>$ \\
\hline & days $* 90$ days 45 & -0.092 & 0.445 & 0.190 \\
\hline
\end{tabular}

* Paired t-Test

\section{Discussion}

Various studies have revealed a remarkable parallel array of prostaglandins, cytokines, cell types, and reactive oxygen species in chronic periodontitis and RA [22, 23].

In this study, the effect of nonsurgical periodontal treatment on RA disease activity and its cross-correlation with inflammatory parameters (CRP and ESR) were examined. A comparison of the results between periods before and after treatment, especially in people with a wide age range, showed a significant improvement. Although few studies have been conducted in this regard, the scope of this research is very broad. In addition, due to differences in the results of several studies, the majority of them being performed on European populations, this study can substantially help to generalize the results of other populations. Despite being a small-scale intervention study, a detailed statistical method was employed to improve the potential of this study.

As shown in the results, periodontitis therapy reduced the disease activity in RA. In a study by Biyıkoğlu et al. (2013), the level of disease activity showed a significant decrease after 1 and 3 months of treatment in 15 patients with chronic periodontitis and RA [24]. In a study conducted by Okada et al. [10] on 55 patients with RA in which the patients were grouped treatment $(\mathrm{n}=26)$ and control $(n=29)$ groups, it was revealed that the treatment group had a greater reduction in DAS28 scores. Thus, it was concluded that, by reducing P. gingivalis, protein citrullination (which is associated with the pathogenesis of RA) is reduced. This is in line with the results of the current study in which the intensity of RA decreased. Smit et al. [25], Erciyas et al. [26], and Oritz [27] also reported higher prevalence rates of periodontitis in patients with RA compared to patients without RA. Furthermore, they reported that RA patients with severe periodontitis showed higher DAS28 scores compared with RA patients with moderate or no periodontitis. Other studies have also approved this association [28-31].

However, the treatment of periodontitis results in the reduction of common inflammatory cytokines and, consequently, reduces the level of RA activity [31-33]. Furthermore, the prevalence of gingivitis is higher in patients with RA than in healthy people; this can be attributed to different factors such as immunology and lack of balance between host response on one hand and periodontal microorganisms on the other. Therefore, with the management and treatment of periodontitis, the inflammatory and immunological effects of periodontal microorganisms in RA can be prevented and reduced [32,33].

The cross-reaction of antibodies produced against microorganisms such as hemolytic streptococcus has also been implicated in increasing the intensity of the disease [34]. Theoretically, periodontitis therapy, similar to the current findings, reduces bacteria and subsequent inflammation caused by these microorganisms; as such, their direct (cross-reactive antibodies) and indirect (inflammation caused by microorganisms) effects on the intensity of the disease activity in RA decreases. In addition, IL-1, TNF- $\alpha$, and IL-6 as well as hyper-responsive monocytes, which are associated with the HLA complex, are jointly enhanced in patients with RA and periodontitis. By controlling each of these diseases, however, the level of these molecules in the body is reduced [35]. Other studies have also emphasized the role of IL-17 in periodontitis and RA [36], 
and periodontitis has been shown to have a synergistic relationship with TNF- $\alpha$ and IL-1. IL-17 is a homodimeric glycosylated polypeptide that is produced by a new subset of CD14 \pm CD14 called TH17 and can be found at high levels in GCF of periodontitis [37].

According to the above facts, regarding the relatively easier and faster periodontitis therapy, it seems that the treatment of periodontitis in patients with RA can reduce inflammation and disease activity in RA. In fact, this does not imply continuous treatment, because the results of the present study showed that long-term follow up after the treatment of periodontitis (90 days as compared to 45 days) had no effect on the severity of RA. This finding is also in accordance with the results of Pinho et al. [30]. Thus, this result could be attributed to periodontitis recurrence and environmental factors such as tobacco smoking and type of hygiene. On the other hand, prolonged use of methotrexate and cyclosporine in RA patients can cause susceptibility to stomatitis and gingival overgrowth [32].

In this study, ESR values were lower at 45 and 90 days after treatment than at baseline. Also, serum CRP levels were lower at 90 days after treatment than at 40 days. However, there were no significant differences between the different time points. The results of previous studies have shown that there is no specific inflammatory indicator for predicting the association between RA and periodontitis. Laboratory markers such as CRP, ESR, arthritis-rheumatoid factor, prostaglandins, and compounds which result from the destruction of collagen changed under all inflammatory conditions. Since there is no significant relationship between these markers and RA activity, it is necessary to consider a series of clinical, immunopathological, and microbiological factors in order to achieve an acceptable recognition [38].

This study has some limitations. The effect of periodontal treatment on the activity of RA was evaluated only in three stages (baseline, 45 days, and 90 days after treatment) with no control on the environmental conditions affecting the values and laboratory variables in order to achieve reliable results. The lack of a control group and the impossibility of assessing other factors, such as tobacco smoking and oral hygiene, are the other limitations of this study.

Therefore, it is recommended that the effect of treatment on periodontitis using other factors such as plaque index, mean pocket depth, and papillary bleeding index and their correlations with the intensity of activity in RA should be evaluated so as to improve the accuracy of the study.

\section{Conclusion}

This study showed significant decreases in RA disease activity levels (DAS28) after non-surgical periodontal treatment. The pathophysiology underlying this finding appears not to be through the alteration of ESR or CRP alone. Therefore, routine dental examinations are recommended for RA patients, so as to reduce dental and oral problems while reducing rheumatic symptoms and RA severity. The certainty of these results needs further studies with regards to the mentioned limitations.

\section{Acknowledgments}

This study was supported by Semnan University of Medical Sciences (grant number: 911), Semnan, Iran.

\section{Conflict of Interest}

The authors declare that they have no conflicts of interest. Nasibeh Alebooyeh declares that she has no conflicts of interest. Jamileh Moghimi declares that she has no conflicts of interest. Amir Hasani declares that he has no conflicts of interest. Raheb Ghorbani declares that he has no conflicts of interest. 


\section{References}

1. Fauci AS. Harrison's principles of internal medicine McGraw-Hill Medical New York; 2008.

2. Pincus T, Callahan L. Reassessment of twelve traditional paradigms concerning the diagnosis, prevalence, morbidity and mortality of rheumatoid arthritis Scand J Rheumatol Suppl. 1989;18(S79):67-96. doi: 10.3109/03009748909092614.

3. Scott D, Coulton B, Symmons D, Popert A. Long-term outcome of treating rheumatoid arthritis: results after 20 years. Lancet. 1987;329(8542):1108-11. doi: 10.1016/ s0140-6736(87)91672-2.

4. Silman AJ, Pearson JE. Epidemiology and genetics of rheumatoid arthritis. Arthritis Res. 2002;4(Suppl 3):S265-S72. doi: 10.1186/ar578

5. Carmona L, Villaverde V, Hernández-García C, Ballina J, Gabriel R, Laffon A. The prevalence of rheumatoid arthritis in the general population of Spain. Rheumatology (Oxford). 2002;41(1):88-95. doi: 10.1093/rheumatology/41.1.88.

6. Rilse T, Jacobsen BK, Gran J. Incidence and prevalence of rheumatoid arthritis in the county of Troms, northern Norway. J Rheumatol. 2000;27(6):1386.

7. Uhlig T, Kvien TK. Is rheumatoid arthritis disappearing? Ann Rheum Dis. 2005;64(1):7-10. doi: 10.1136/ ard.2004.023044.

8. van der Helm AH, Wesoly JZ, Huizinga TW. Understanding the genetic contribution to rheumatoid arthritis. Curr Opin Rheumatol. 2005;17(3):299-304. doi: 10.1097/01. bor.0000160780.13012.be.

9. Begovich AB, Carlton VE, Honigberg LA, Schrodi SJ, Chokkalingam AP, Alexander HC, et al. A missense single-nucleotide polymorphism in a gene encoding a protein tyrosine phosphatase (PTPN22) is associated with rheumatoid arthritis. Am J Hum Genet. 2004;75(2):330-7. doi: $10.1086 / 422827$.

10. Okada M, Kobayashi T, Ito S, Yokoyama T, Abe A, Murasawa $\mathrm{A}$, et al. Periodontal treatment decreases levels of antibodies to Porphyromonas gingivalis and citrulline in patients with rheumatoid arthritis and periodontitis. J periodontol. 2013;84(12):e74-e84. doi: 10.1902/ jop.2013.130079.

11. Mikuls TR. Help stop tooth decay... and prevent RA? J Rheumatol. 2010;37(6):1083-5. doi: 10.3899/ jrheum. 100173.

12. Rosenstein ED, Greenwald RA, Kushner LJ, Weissmann G. Hypothesis: the humoral immune response to oral bacteria provides a stimulus for the development of rheumatoid arthritis. Inflammation. 2004;28(6):311-8. doi: 10.1007/s10753-004-6641-z.

13. Marotte H, Farge P, Gaudin P, Alexandre C, Mougin B, Miossec $\mathrm{P}$. The association between periodontal disease and joint destruction in rheumatoid arthritis extends the link between the HLA-DR shared epitope and severity of bone destruction. Ann Rheum Dis. 2006;65(7):905-9. doi: 10.1136/ard.2005.036913.
14. de Pablo P, Dietrich T, McAlindon TE. Association of periodontal disease and tooth loss with rheumatoid arthritis in the US population. J Rheumatol. 2008;35(1):70-6.

15. Mikuls TR, Payne JB, Reinhardt RA, Thiele GM, Maziarz E, Cannella AC, et al. Antibody responses to Porphyromonas gingivalis (P. gingivalis) in subjects with rheumatoid arthritis and periodontitis. Int Immunopharmacol. 2009;9(1):38-42. doi: 10.1016/j.intimp.2008.09.008.

16. Genco RJ. Current View of Risk Factors for Periodontal Diseases*. J Periodontol. 1996;67(10s):1041-9. doi: 10.1902/jop.1996.67.10s.1041.

17. Mercado F, Marshall RI, Klestov AC, Bartold PM. Is there a relationship between rheumatoid arthritis and periodontal disease? J Clin Periodontol. 2000;27(4):267-72. doi: 10.1034/j.1600-051x.2000.027004267.x.

18. Mercado F, Marshall RI, Klestov A, Bartold P. Relationship between rheumatoid arthritis and periodontitis. J Periodontol. 2001;72(6):779-87. doi: 10.1902/ jop.2001.72.6.779.

19. Wells G, Becker JC, Teng J, Dougados M, Schiff M, Smolen J, et al. Validation of the 28-joint Disease Activity Score (DAS28) and European League Against Rheumatism response criteria based on C-reactive protein against disease progression in patients with rheumatoid arthritis, and comparison with the DAS28 based on erythrocyte sedimentation rate. Ann Rheum Dis. 2009;68(6):954-60. doi: 10.1136/ard.2007.084459.

20. Salaffi F, Cimmino M, Leardini G, Gasparini S, Grassi W. Disease activity assessment of rheumatoid arthritis in daily practice: validity, internal consistency, reliability and congruency of the Disease Activity Score including 28 joints (DAS28) compared with the Clinical Disease Activity Index (CDAI). Clin Exp Rheumatol. 2009;27(4):552-9.

21. Van Gestel AM, Haagsma CJ, Van Riel PLCM. Validation of rheumatoid arthritis improvement criteria that include simplified joint counts. Arthritis Rheum. 1998;41(10):1845-50. doi:

22. B1yıkog `u B, Buduneli N, Kardes, ler L, Aksu K, O* der G, Ku"tu"kc,u"ler N. Evaluation of t-PA, PAI-2, IL$1 \mathrm{~b}$ and PGE2 in gingival crevicular fluid of rheumatoid arthritis patients with periodontal disease. J Clin Periodontol. 2006;33(9):605-611. doi: 10.1111/j.1600051x.2006.00961.x.

23. Biyıkog lu B, Buduneli N, Kardes, ler L, Aksu K, Pitkala M, Sorsa T. MMP-8, MMP-13 and TIMP-1 levels in gingival crevicular fluid of rheumatoid arthritis patients with inflammatory periodontal disease. J Periodontol. 2009;80(8):1307-1314. doi: 10.1902/jop.2009.090130. $10.1002 / 1529-0131(199810) 41: 10<1845:$ :aidart17>3.3.co;2-b.

24. Bıyıkoğlu B, Buduneli N, Aksu K, Nalbantsoy A, Lappin DF, Evrenosoğlu E, et al. Periodontal therapy in chronic periodontitis lowers gingival crevicular fluid interleukin-1beta and DAS28 in rheumatoid arthritis patients. Rheumatol Int. 2013;33(10):2607-16. doi: 10.1007/ 
s00296-013-2781-5.

25. Smit M, Westra J, Vissink A, Doornbos-van der Meer B, Brouwer E, van Winkelhoff AJ. Periodontitis in established rheumatoid arthritis patients: a cross-sectional clinical, microbiological and serological study. Arthritis Res Ther. 2012;14(5):R222. doi: 10.1186/ar4061.

26. Erciyas K, Sezer U, Üstün K, Pehlivan Y, Kisac1k B, Şenyurt S, et al. Effects of periodontal therapy on disease activity and systemic inflammation in rheumatoid arthritis patients. Oral Dis. 2013;19(4):394-400. doi: 10.1111/ odi.12017.

27. Ortiz P, Bissada N, Palomo L, Han Y, Al-Zahrani M, Panneerselvam A, et al. Periodontal therapy reduces the severity of active rheumatoid arthritis in patients treated with or without tumor necrosis factor inhibitors. J Periodontol. 2009;80(4):535-40. doi: 10.1902/jop.2009.080447.

28. Berthelot JM, Le Goff B. Rheumatoid arthritis and periodontal disease. Joint Bone Spine. 2010; 77(6): 537-41. doi: 10.1016/j.jbspin.2010.04.015.

29. Soory M. Hormone mediation of immune responses in the progression of diabetes, rheumatoid arthritis and periodontal diseases. Curr Drug Targets Immune Endocr Metabol Disord. 2002; 2(1): 13-25. doi: $10.2174 / 1568008024606310$.

30. Pinho MN, Oliveira RD, Novaes AB Jr, Voltarelli JC. Relationship between periodontitis and rheumatoid arthritis and the effect of non-surgical periodontal treatment. Braz Dent J. 2009; 20(5):355-64. doi: 10.1590/s010364402009000500001 .
31. Bartold PM, Marshall RI, Haynes DR. Periodontitis and rheumatoid arthritis: A Review. J Periodontol. 2005; 76(11): 2066-74. doi: 10.1902/jop.2005.76.11-s.2066.

32. Mercado FB, Marshall RI, Klestov AC, Bartold PM. Relationship between rheumatoid arthritis and periodontitis. J Periodontol. 2001; 72(6): 779-87. doi: 10.1902/ jop.2001.72.6.779.

33. Sjöström L, Laurell L, Hugoson A, Håkansson JP. Periodontal conditions in adults with rheumatoid arthritis. Community Dent Oral Epidemiol. 1989; 17(5): 234-6. doi: 10.1111/j.1600-0528.1989.tb00623.x.

34. Neville BW, Damm DD, Allen CM, Bouquot J. Oral and maxillofacial pathology. 3rd ed. Philadelphia: Saunders/ Elsevier; 2009: 877-88.

35. Miranda LA, Fischer RG, Sztajnbok FR, Figueredo CM, Gustafsson A. Periodontal conditions in patients with juvenile idiopathic arthritis. J Clin Periodontol. 2003; 30(11): 969-74. doi: 10.1034/j.1600-051x.2003.00406.x.

36. Lubberts E. The role of IL-17 and family members in the pathogenesis of arthritis. Curr Opin Investig Drugs. 2003; 4(5): 572-577.

37. Paradowska A, Maślińiski W, Grzybowska-Kowalczyk A, Łacki J. The function of interleukin 17 in the pathogenesis of rheumatoid arthritis. Arch Immunol Ther Exp (Warsz). 2007; 55(5): 329-334. doi: 10.1007/s00005-007-0032-8.

38. Ishi EP, Bertolo MB, Rossa C, Jr., Kirkwood KL, Onofre MA. Periodontal condition in patients with rheumatoid arthritis. Braz Oral Res. 2008; 22(1): 72-7. doi: 10.1590/ s1806-83242008000100013. 